\title{
Frontal processing and auditory perception
}

\author{
Timothy D. Griffiths, $, 2,3$, CA Virginia Penhune, ${ }^{4}$ Isabelle Peretz, ${ }^{5}$ Jenny L. Dean, ${ }^{2}$ Roy D. Patterson ${ }^{6}$ \\ and Gary G. R. Green ${ }^{2}$
}

\begin{abstract}
Departments of ' Neurology and ${ }^{2}$ Physiological Sciences, Newcastle University Medical School, Framlington Place, Newcastleupon-Tyne NE2 4HH; ${ }^{3}$ Wellcome Department of Cognitive Neurology, Institute of Neurology, London WCIN 3BG, UK; ${ }^{4}$ McConnell Brain Imaging Centre, Montreal Neurological Institute, Montreal H3A 2B4; ${ }^{5}$ Départment de Psychologie, Université de Montréal, Montréal H3C 3J7, Canada; ${ }^{6}$ Centre for the Neural Basis of Hearing, Physiology Department, University of Cambridge, Cambridge CB2 3EG, UK

$\mathrm{CA}, 2^{2}$ Corresponding Author and Address
\end{abstract}

Received 5 January 2000; accepted 15 January 2000

Acknowledgements: We thank I.R. This collaboration was made possible by grants to T.D.G. from the Wellcome Trust (UK) and to I.P. from the Medical Research Council of Canada.

Disordered processing of the pattern in sound over time has been observed in a number of clinical disorders, including developmental dyslexia. This study addresses the brain mechanisms required for the perception of such a pattern. We report the systematic evaluation of temporal perception in a patient with a single intact right auditory cortex and a large right frontal lobe lesion. A striking dissociated deficit was demonstrated in the perception of temporal pattern at the level of tens or hundreds of milliseconds. This proves that, contrary to common belief, mechanisms in the pathway up to and including the primary auditory cortex are not sufficient for the normal perception of temporal pattern. This work suggests a need for frontal processing for the normal perception of auditory pattern. NeuroReport 1 1:919-922 (C) 2000 Lippincott Williams \& Wilkins.

Key words: Auditory; Cortex; Human; Perception; Psychophysics

\section{INTRODUCTION}

Abnormal processing of the patterns in sound over time has been suggested in a number of clinical disorders, including receptive language disorders in children and developmental dyslexia [1-4]. Examples of such patterns are continuous changes in sound properties such as frequency or amplitude (called frequency or amplitude modulation). Animal neurophysiology [5-7] and human functional imaging [8] has demonstrated neural mechanisms in the ascending auditory pathway and auditory cortex for the processing of frequency and amplitude modulation. In this study we tested the hypothesis that such mechanisms are adequate for the perception of temporal pattern. Specifically, we investigated whether additional processing in the frontal cortex is necessary for the normal perception of auditory temporal pattern. Frontal processing has been suggested to be necessary for normal visual perception [9].

We have been allowed to test this hypothesis by studying a patient who is unable to carry out normal frontal processing of auditory stimuli. Such patients are rare; abnormal frontal lobe processing of auditory stimuli requires either bilateral lesions of the frontal cortex, or destruction of the auditory cortex on one side and a lesion anterior to the auditory cortex on the other side. We have assessed the perception of the temporal structure of sound at a number of levels, from the millisecond level to the hundreds of milliseconds level. This allows the demonstration of dissociated perceptual deficits for sounds with different temporal patterns.

\section{PATIENT AND METHODS}

Subject IR sustained bilateral brain damage 10 years before assessment (Fig. 1) during surgery for bilateral middle cerebral artery aneurysms. The extent of her lesions was compared with a probabilistic map of the primary auditory cortex [10]. In the left hemisphere most of the superior temporal gyrus is destroyed, including the primary auditory area within Heschl's gyrus (HG). In the right hemisphere the damage to the temporal lobe involves the superior temporal lobe anterior to $\mathrm{HG}$, in addition to a large frontal lesion involving most of the precentral and inferior frontal gyri. HG is spared, as are the planum temporale posterior to it and lateral cortex in the superior temporal planum. There is no damage to the brain stem or thalamus. Auditory function in IR is therefore subserved by a single intact pathway up to and including the right primary auditory cortex, that is isolated from anterior areas in the temporal and frontal lobes.

Psychoacoustic assessment was carried out over $60 \mathrm{~h}$ with the patient's informed consent. Assessment included audiometry and measurement of the perception of the 


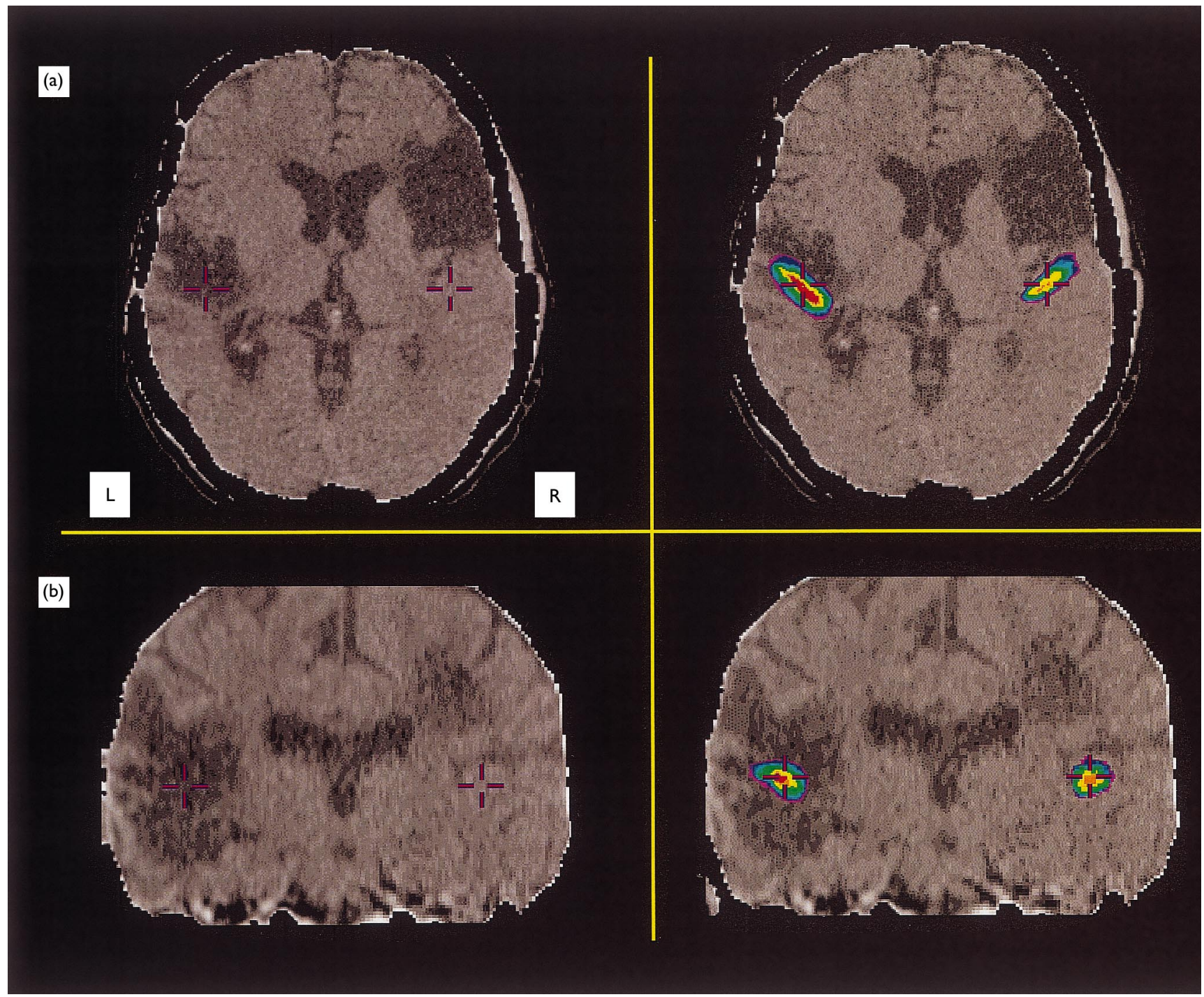

Fig. I. High resolution CT scan (1.5 mm slices) of subject I.R. (age 42). MRI scanning was not performed due to ferrous aneursm clips applied to bilateral middle cerebral artery territory aneurysms. CT is transformed into the stereotaxic space of Talairach, and co-registered with the probabilistic map of Heschl's gyrus (HG). (a) Axial slices at $z=7 \mathrm{~mm}$, shown alone and co-registered with the probabilistic map (scaled from $25-100 \%$ probability). (b) Coronal slices at $x=-19 \mathrm{~mm}$. Cursor indicates the same position in standardized space in both images. Complete destruction of left HG and complete sparing of right HG is demonstrated. Extensive right frontal damage is also evident in these sections.

temporal structure at a number of levels. The ability to perceive the fine temporal structure in sounds was assessed using iterated rippled noise (IRN [11]). This stimulus is perceived as a noise with a pitch, the perceptual properties of which parallel the temporal regularity in the stimulus at the millisecond level. The ability to perceive the temporal structure at the level of tens or hundreds of milliseconds was assessed by measuring the binaural detection of sinusoidal amplitude modulation (AM) and sinusoidal frequency modulation (FM) of a $500 \mathrm{~Hz}$ carrier. Modulation rates of $2 \mathrm{~Hz}$ (corresponding to temporal structure at the level of hundreds of milliseconds) and $40 \mathrm{~Hz}$ (corresponding to temporal structure at the level of tens of milliseconds) were investigated.

Fig. 2. Psychometric functions for subject I.R. The horizontal lines show the $95 \%$ confidence interval for the threshold for eight naive normal individuals aged $3 \mathrm{I}-4 \mathrm{I}$. This allows comparison with the $75 \%$ threshold value for I.R. (a) Detection of iterated rippled noise (IRN) as a function of gain; passband $\mathrm{I}-4 \mathrm{kHz}$, delay of $10 \mathrm{~ms}$, eight iterations, gain varied between $0.0 \mathrm{I}$ and 0.32 in octave steps. Binaural (bin) and monaural (mon) detection is shown. I.R. has a normal threshold at the left ear, the predominant input to the intact right auditory cortex. (b) Detection of binaural sinusoidal amplitude modulation (AM) at modulation rates of $2 \mathrm{~Hz}$ and $40 \mathrm{~Hz}$ (carrier frequency $500 \mathrm{~Hz}$ ). (c) Detection of binaural sinusoidal frequency modulation (FM) at rates of $2 \mathrm{~Hz}$ and $40 \mathrm{~Hz}$ (carrier frequency $500 \mathrm{~Hz}$ ). 

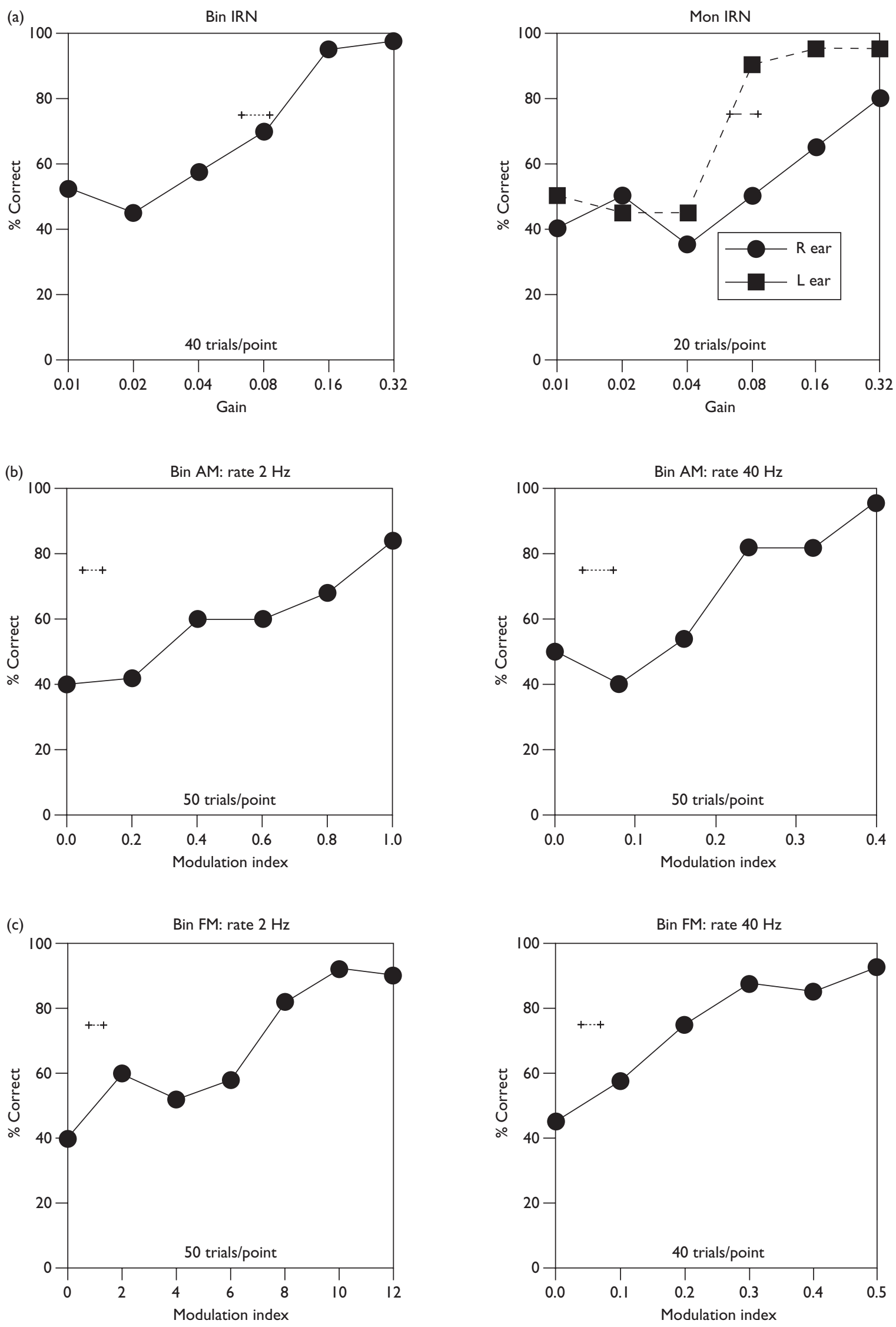
Perception of all temporal stimuli was measured using a two-alternative-forced-choice technique. Pairs of sounds of $1 \mathrm{~s}$ duration were presented over headphones at a sensation level of $60 \mathrm{~dB}$. Synchronous masking noise at a sensation level of $35 \mathrm{~dB}$ was used in the other ear for the monaural tasks. The subject was required to detect which of two intervals contained the target sound. The target interval and signal-to-noise ratio level were varied randomly from trial to trial. Psychometric functions were plotted to allow determination of the threshold at which $75 \%$ correct detection was achieved. The functions were all based on at least 20 trials per point to allow a reliable estimate of threshold. The $75 \%$ threshold estimates were obtained from Weibull functions [12] fitted to the data using an automated algorithm. The threshold data were compared to control data from eight age-matched naive subjects.

\section{RESULTS}

Pure tone audiometry was normal. Testing of the monaural perception of temporal structure at the millisecond level using IRN (Fig. 2a) showed that this was normal for the left ear, which is the predominant input to the preserved right auditory cortex. In contrast, her ability to detect temporal structure at the level of tens of milliseconds and above was markedly abnormal (Fig. $2 b, c)$. At a modulation rate of $2 \mathrm{~Hz}$, corresponding to temporal structure at the level of hundreds of milliseconds, detection thresholds for AM and FM were 11.0 and 6.4 times normal respectively ( $p<0.001$ for both comparisons, one sample $t$-test). At a modulation rate of $40 \mathrm{~Hz}$, corresponding to temporal structure at the level of tens of milliseconds, detection thresholds for AM and FM were 4.6 and 4.1 times normal respectively $(p<0.001$ for both comparisons, one sample $t$ test). Monaural testing of AM and FM did not show systematic differences between the ears as for IRN. Previous analysis of the detection of temporal pattern by IR, in segmented rather than continuous sounds, also demonstrated a striking deficit at similar levels of temporal structure to those that revealed the modulation deficits $[13,14]$.

\section{DISCUSSION}

The deficit in the analysis of modulated sound in this patient is a perceptual disorder, and is not due to deafness, or a deficit in attention or working memory. The normal detection of IRN required similar selective attention to simple acoustic features using similar two-alternative forced-choice psychophysics, and similar working memory demands for those features. This study demonstrates preserved perception of pure tones and the pitch of IRN in a subject with an intact pathway up to and including the primary auditory cortex. This is consistent with the representation of spectral and fine temporal structure in human primary auditory cortex being a neural correlate of conscious perception [15]. Such representation has been demonstrated by previous human studies using magnetoencephalography [16,17]. Alternatively, we cannot dismiss the possibility that preserved areas lateral and posterior to HG might be required for the perception of pitch. The lateralisation of performance for IRN pitch perception suggests that this process depends on processing in the medial geniculate body or auditory cortex; structures at lower points in the ascending pathway exhibit extensive cross connections. The involvement of the thalamus or cortex in perceptual processing would not preclude detection of fine temporal structure earlier in the pathway.

These data indicate that the normal perception of temporal structure above the millisecond range cannot be subserved by an intact pathway up to and including the right primary auditory cortex. Mechanisms for modulation analysis in this pathway [5-8] are not, therefore, sufficient for normal modulation perception in man. What, then, is the sufficient substrate for higher-order temporal perception? We hypothesise that connection between the auditory cortices and the frontal cortex is necessary for the human perception of continuous temporal modulation in sounds. Specifically, we hypothesise that the critical connection is between secondary auditory cortex and frontal cortex. Immediately anterior to the primary auditory area in the macaque is a secondary auditory area (rostral area [18]). This area projects to the frontal cortex, and may have a specific role in temporal pattern processing [19]. If a homologous area were to exist in humans, the lesion in IR would be more likely to affect its frontal connections than the area itself; the lesion is well anterior to $\mathrm{HG}$ in the temporal lobe, and involves large areas of the frontal cortex.

This work proves that the disordered perception of temporal pattern observed in certain clinical disorders need not necessarily be due to an abnormality in the ascending auditory pathway or auditory cortex.

\section{REFERENCES}

1. Griffiths TD, Rees A and Green GGR. Neurocase 5, 365-378 (1999).

2. Stefanatos GA, Green GGR and Ratcliff GG. Arch Neurol 46, 871-875 (1989).

3. Witton C, Talcott JB, Hansen PC et al. Curr Biol 8, 791-797 (1998).

4. Baldeweg T, Richardson A, Watkins S et al. Ann Neurol 45, 495-503 (1999).

5. Rees A and Moller AR. Hear Res 10, 301-330 (1983).

6. Langner G and Schreiner CE. J Neurophysiol 60, 1799-1822 (1988).

7. Whitfield IC and Evans EF. J Neurophysiol 28, 655-672 (1965).

8. Harms MP, Melcher JR and Weisskoff R. Neuroimage 7, S365 (1998).

9. Crick F and Koch C. Nature 375, 121-123 (1995).

10. Penhune VB, Zatorre RJ, MacDonald JD and Evans AC. Cerebr Cortex 6 , 661-672 (1996).

11. Griffiths TD, Buechel C, Frackowiak RSJ and Patterson RH. Nature Neurosci 1, 421-427 (1998).

12. MacMillan NA and Creelman CD. Detection Theory: A User's Guide. Cambridge: Cambridge University Press, 1991.

13. Peretz I and Gagnon L. Neurocase 5, 21-30 (1999).

14. Patel AD, Peretz I, Tramo M and Labreque R. Brain Lang 61, 123-144 (1998).

15. Frith C, Perry R and Lumer E. Trends Cogn Sci 3, 105-114 (1999).

16. Pantev C, Hoke M, Lutkenhoner B and Lehnertz K. Science 242, 486-488 (1989).

17. Langner G, Sams M, Heil P and Schulze H. J Comp Physiol 181, 665-676 (1997).

18. Merzenich MM and Brugge JF. J Neurophysiol 24, 193-202 (1973).

19. Rauschecker JP. Audiol NeuroOtol 3, 86-103 (1998). 